\title{
Correction to: Bonded contact and general crack problems in generalized materials and their relationships
}

\section{I. Fabrikant}

Published online: 20 January 2020

(C) Springer Nature B.V. 2020

Correction to: Meccanica (2018) 53:2709-2723

https://doi.org/10.1007/s11012-018-0857-8

Due to an unfortunate turn of events, Eqs. 52, 155, 156 and 157 contain errors. Please find below the correct version of the equations mentioned that should be regarded as the final version by the reader.

$$
\begin{aligned}
& \int_{S} \int K_{31}^{0}\left(x_{1}-x_{10}, x_{2}-x_{20}\right) u_{10}\left(x_{10}, x_{20}\right) d x_{10} d x_{20} \\
& \quad+\int_{S} \int K_{32}^{0}\left(x_{1}-x_{10}, x_{2}-x_{20}\right) u_{20}\left(x_{10}, x_{20}\right) d x_{10} d x_{20} \\
& \quad+\int_{S} \int K_{33}^{0}\left(x_{1}-x_{10}, x_{2}-x_{20}\right) u_{30}\left(x_{10}, x_{20}\right) d x_{10} d x_{20}=\sigma_{33},
\end{aligned}
$$

The original article can be found online at https:// doi.org/10.1007/s11012-018-0857-8.

\section{I. Fabrikant $(\bowtie)$}

Prisoner \#167932D, Archambault Jail, 242 Montee Gagnon, Ste-Anne-des-Plaines, QC J0N1H0, Canada e-mail: valery_fabrikant@hotmail.com

$$
\begin{aligned}
& \int_{-\infty}^{\infty} \int_{-\infty}^{\infty} \frac{\xi_{1}^{2}}{\left(\xi_{1}^{2}+\xi_{2}^{2}\right)^{3 / 2}} \exp \left(-\zeta x_{3}\right) \\
& \quad \times \exp \left[-i\left(x_{1} \xi_{1}+x_{2} \xi_{2}\right)\right] d \xi_{1} d \xi_{2} \\
& \quad=\pi\left[\frac{1}{R_{0}}+\frac{x_{2}^{2}-x_{1}^{2}}{R_{0}\left(R_{0}+z\right)^{2}}\right], \\
& \int_{-\infty}^{\infty} \int_{-\infty}^{\infty} \frac{\xi_{2}^{2}}{\left(\xi_{1}^{2}+\xi_{2}^{2}\right)^{3 / 2}} \exp \left(-\zeta x_{3}\right) \\
& \quad \times \exp \left[-i\left(x_{1} \xi_{1}+x_{2} \xi_{2}\right)\right] d \xi_{1} d \xi_{2} \\
& \quad=\pi\left[\frac{1}{R_{0}}-\frac{x_{2}^{2}-x_{1}^{2}}{R_{0}\left(R_{0}+z\right)^{2}}\right], \\
& \int_{-\infty}^{\infty} \int_{-\infty}^{\infty} \frac{\xi_{1} \xi_{2}}{\left(\xi_{1}^{2}+\xi_{2}^{2}\right)^{3 / 2}} \exp \left(-\zeta x_{3}\right) \\
& \times \exp \left[-i\left(x_{1} \xi_{1}+x_{2} \xi_{2}\right)\right] d \xi_{1} d \xi_{2}=-\frac{2 \pi x_{1} x_{2}}{R_{0}\left(R_{0}+z\right)^{2}}
\end{aligned}
$$

Publisher's Note Springer Nature remains neutral with regard to jurisdictional claims in published maps and institutional affiliations. 